Article

\title{
Tunable Optical Bistability, Tristability and Multistability in Arrays of Graphene
}

\author{
Xiuju Zhao ${ }^{1,+}$, Bin $\mathrm{Xu}^{2, \dagger}{ }^{+}$, Xiangna Kong ${ }^{2,+}$, Dong Zhong ${ }^{2}$, Ming Fang ${ }^{2, *}$ and Dong Zhao ${ }^{2, *} \mathbb{C}$ \\ 1 School of Mathematics and Statistics, Hubei University of Arts and Science, Xiangyang 441053, China; \\ 10719@hbuas.edu.cn \\ 2 Laboratory of Functional Materials and Devices for Informatics, School of Education, School of Computer \\ Science and Technology, Hubei University of Science and Technology, Xianning 437100, China; \\ jwcxb@hbust.edu.cn (B.X.); kongxn@hbust.edu.cn (X.K.); zhongdong@hbust.edu.cn (D.Z.) \\ * Correspondence: fangming@hbust.edu.cn (M.F.); zhaodong@hbust.edu.cn (D.Z.) \\ + These authors contributed equally.
}

Received: 18 July 2020; Accepted: 18 August 2020; Published: 20 August 2020

check for updates

\begin{abstract}
The optical bistability, tristability and multistability are explored in arrays of graphene. The arrays are periodically arranged spatially by single sheets of graphene. Optical bistability could be achieved with a strong enough incident intensity of light wave. The thresholds of optical bistability and the intervals between the upper and lower thresholds change with the surface conductivity of graphene and the incident wavelength. By increasing the intensity of incident light, tristability and multistability can be induced as well. Furthermore, the thresholds of bistability, tristability and multistability can be regulated via the chemical potential of graphene. This study may have potential applications in optical logic gates, all-optical switches and photomemory.
\end{abstract}

Keywords: nonlinearity; graphene; array; optical bistability; all-optical switch

\section{Introduction}

Booming all-optical communication has revolutionized information transmission technology drastically, and in the all-optical communicating network, the all-optical switch is one of the key types of components. A typical all-optical switch could be realized by optical bistability, tristability or multistability effects, in which any given value of input intensity can correspond to two, three or multiple stable output states, respectively [1]. Optical bistability, tristability and multistability can also be utilized for optical memory [2], sensors [3] or logic gates [4]. Although the hysteresis phenomena originate from magnetic effects, investigations demonstrate that bistable effects are widespread in optics [5], thermotics [6], and mechanics [7,8]. The Fabry-Perot cavity is the simplest structure to realize optical bistability [9]. Recently, Fano resonance [10] and surface plasmon polaritons (SPPs) [11] have been proposed to achieve optical bistability too. The common characteristic of those systems is that they can localize the light field and enhance the third-order nonlinear effect of materials. Localization of light field can be also observed in many other constructions or effects, such as defective photonic crystals [12], nanocavity [13], topological bound modes [14-17] and solitons [18,19]. Intense laser light can be utilized, not for optical bistability, but for probing the internal structure of molecules [20-22].

Graphene is an excellent two-dimensional material. It possesses predominant mechanical properties [23,24] and superconductivity [25]. Furthermore, it has a considerable Kerr coefficient [26]. Past research has shown that low-threshold optical bistability and multistability could be achieved through embedding graphene into defective photonic crystals or combining graphene with dielectrics. Moreover, the bistable thresholds and the intervals between the lower threshold and upper threshold could be regulated conveniently by the external voltage on graphene. 
Since single sheets of graphene are arrayed periodically to form one-dimensional photonic crystals, there are photonic bandgaps in wave-vector space, similar to the semiconductor bands structure. Explorations prove that arrays of graphene sheets could significantly localize the light field and optical solitons may arise at the edge of the bandgaps $[27,28]$. The nonlinear optical effects, such as optical bistability, tristability and multistability, all run based on localization enhancement of light field. Therefore, it inspires our interest to explore optical bistability, tristability and multistability in arrays of graphene sheets.

The tunable optical bistability, tristability and multistability are explored here in arrays of graphene. Single sheets of graphene array periodically along the horizontal direction. The linear and nonlinear transmittances are firstly investigated. Then, we give the bistable relationship between the output and input intensity. To illustrate the regulation on bistability by the external voltage, we simulate the surface conductivity of graphene in the parametric space composed of the external voltage and the incident wavelength. By further increasing the incident intensity of light, optical tristability and multistability can also be induced. This research can be applied to all-optical switches and optical storages.

\section{Periodic Graphene Arrays}

Single sheets of graphene are embedded in silicon dioxide to form arrays of graphene with a fixed spatial period as shown in Figure 1. Silicon and silicon dioxide are usually used for waveguides or substrate $[29,30]$. In the arrays, the thickness of dielectrics, viz., silicon dioxide, is noted by $d$ and $N$ is the period number of graphene. Graphene is an ultrathin two-dimensional material equipped with distinct electrical conductivity. Its surface conductivity is governed by the Kubo formula [1,31]:

$$
\sigma_{1}\left(\omega, \mu_{c}, \tau, T\right)=-\frac{i e\left(\omega+i \tau^{-1}\right)^{2}}{\pi h^{2}}\left[\int_{-\infty}^{+\infty} \frac{|\varepsilon|}{\left(\omega+i \tau^{-1}\right)^{2}} \frac{\partial f_{d}(\varepsilon)}{\partial \varepsilon} d \varepsilon-\int_{0}^{+\infty} \frac{\partial f_{d}(-\varepsilon)-\partial f_{d}(\varepsilon)}{\left(\omega+i \tau^{-1}\right)^{2}-4(\varepsilon / h)^{2}} d \varepsilon\right],
$$

where $f_{d}=1 /\left(1+\exp \left[\left(\varepsilon-\mu_{c}\right) /\left(k_{B} T\right)\right]\right)$ is the Fermi-Dirac statistical distribution, $k_{B}$ denotes the Boltzmann constant, $\tau$ is the momentum delay time, $i$ is the unit of imaginary number, $\varepsilon$ denotes the particle energy, $\mu_{c}$ represents the chemical potential of graphene (also known as the Fermi level $E_{F}$ ), and $T$ is the temperature, $e$ is the unit charge of electron. The first term of Equation (1) represents the intraband electron-photon scattering process, which can be obtained after integration.

$$
\sigma_{\text {intra }}=i \frac{e^{2} k_{B} T}{\pi h^{2}}\left\{\frac{\mu_{c}}{k_{B} T}+2 \ln \left[\exp \left(-\frac{\mu_{c}}{k_{B} T}\right)+1\right]\right\} .
$$

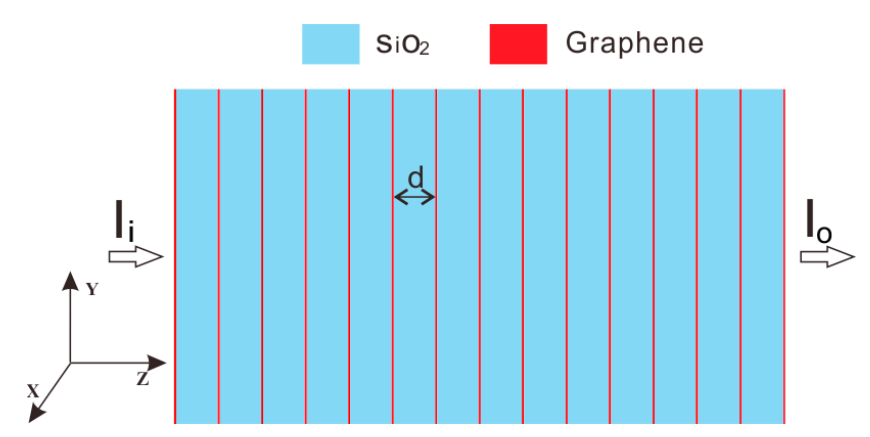

Figure 1. Graphene arrays constructed from monolayer graphene. The substrate material is silicon dioxide. Graphene is arranged periodically, where the thickness of $\mathrm{SiO}_{2}$ is $d=10 \mathrm{~nm}$. The equivalent thickness of a graphene sheet is $\Delta=0.3 \mathrm{~nm}$. 
The second term of Equation (1) represents the interband transition of electrons. For $\hbar \omega,\left|\mu_{c}\right| \gg$ $k_{B} T$, it can be simplified as

$$
\sigma_{\text {inter }}=i \frac{e^{2}}{4 \pi h^{2}} \ln \left[\frac{2\left|\mu_{c}\right|-h\left(\omega+i \tau^{-1}\right)}{2\left|\mu_{c}\right|+h\left(\omega+i \tau^{-1}\right)}\right] .
$$

The energy band of intrinsic graphene is provided in Figure 2a. Graphene exhibits strong broadband absorption which is 50 times higher than GaAs. For graphene, its surface conductivity is contributed by interband and intraband transitions, which are related to the frequency of photons and the chemical potential on graphene $\mu_{c}=E_{F}$. The chemical potential of doped graphene can be controlled by chemical doping or external gate voltage [32]. For intrinsic graphene $E_{F}=0$, there is only interband transition and no intraband contribution. Figure $2 \mathrm{~b}$ gives the transitions of electrons in graphene for $E_{F}>0$. The Fermi level is elevated as a negative gate voltage bias on graphene. The effect of this operation is similar to the case of $n$-doping. Interband transition only occurs as the incident photon satisfies the condition $\hbar \omega_{1}>2\left|E_{F}\right|$, so the low-wavelength dynamic surface conductivity is dominated by interband contribution. In the $\mathrm{THz}$ band, the energy of the photon is relatively low. Interband transition dominates for $\hbar \omega_{2}<2\left|E_{F}\right|$ and the conductivity of graphene is approximately in the form of a Drude model, similar to that of the metals. As the graphene is biased by a positive gate voltage, similar to the case of $p$-doped, the Fermi level degrades $E_{F}<0$ as shown in Figure 2c. Interband transition occurs above the threshold of $\hbar \omega_{1}>2\left|E_{F}\right|$ as well.

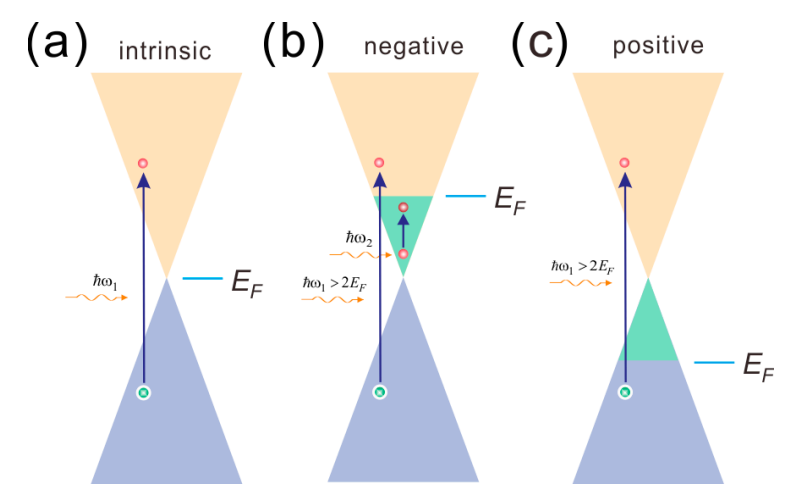

Figure 2. (a) Schematic illustration of interband transitions in intrinsic graphene. $(\mathbf{b}, \mathbf{c})$ Intraband and interband transitions with a negative bias voltage and a positive bias voltage, respectively.

Graphene could be viewed as an equivalent dielectric and numerous simulations have shown that the deviations caused by such emulating can be negligible as long as the equivalent thickness is less than $1 \mathrm{~nm}[33,34]$.

As a transverse wave is incident in the arrays of graphene from the left and its intensity is low enough, the third-order nonlinear effect in graphene can be ignored. The symbol $I_{0}$ represents the transmitted intensity of light and $I_{i}$ is the intensity incident light wave. The linear transmittance and reflectivity of graphene arrays are derived by the forward transmission matrix method (FTMM) [1,35]. However, the nonlinear effect of graphene cannot be ignored as the incident light is strong enough. Then, we can utilize the inverse transmission matrix method (ITMM) [36] to simulate the nonlinear transmittance in the graphene arrays and the input-output intensity relationship of light.

The nonlinear conductivity coefficient of graphene is denoted by

$$
\sigma_{3}=-i \frac{3}{8} \frac{e^{2}}{\pi h}\left(\frac{e V_{F}}{\mu_{c} \omega}\right)^{2} \frac{\mu_{c}}{\omega}
$$

where $V_{F} \approx c / 300$ is the Fermi velocity $[1,20]$. We divide the dielectric into numerous sublayers and calculate the incident light intensity of each sublayer in reverse through the transmission matrix for different output light intensity. For graphene, the nonlinear modification to the surface conductivity of 
graphene is carried out by using the relationship between the surface conductivity and local intensity of electric field. Then, the complete form of surface conductivity is noted by

$$
\sigma_{g}=\sigma_{1}+\sigma_{3}\left|E_{/ /}\right|^{2}
$$

Since graphene is regarded as a dielectric with a certain thickness, its equivalent dielectric constant is expressed as

$$
\varepsilon_{g}=1+i \frac{\sigma_{1} \eta_{0}}{k_{0} \Delta}+i \frac{\sigma_{3} \eta_{0}}{k_{0} \Delta}\left|E_{/ /}\right|^{2}
$$

where $\Delta$ is the equivalent thickness of graphene, the vacuum resistivity is noted by $\eta_{0}$ and $k_{0}$ represents the incidence wave number in vacuum. Here, we collectively give the parameters as follows: the environment temperature $T=300 \mathrm{~K}$, the equivalent thickness $\Delta=0.3 \mathrm{~nm}$, the thickness of each layer of $\mathrm{SiO}_{2}$ is $d=10 \mathrm{~nm}$, the period number of graphene arrays is $N=40$, the dielectric constant of silicon dioxide is $\varepsilon=2.1$.

\section{Optical Bistability, Tristability and Multistability}

The reflectivity and transmittance are given in Figure 3a as a light impinges upon the arrays of graphene. One can see that there is an upward saltus step on the transmission curve. As the input wavelength is lower than $1.25 \mu \mathrm{m}$, the transmittance $T$ fluctuates around 0.5. While the wavelength is greater than $1.25 \mu \mathrm{m}$, the transmittance approximates to 1 and fluctuates around this value. There are many peaks in transmission spectrum and these maxima in the curve correspond to resonant states. In addition, the reflectivity $R$ is low and fluctuates around zero, indicating that most energy of light has been transmitted. The peaks of transmittance face to the zero points of reflectivity exactly. Interband transition of electrons dominates as a short wavelength is incident in the graphene sheets, inducing large loss in intensity of light wave, while the incident wave with long wavelength mainly triggers intraband transition.
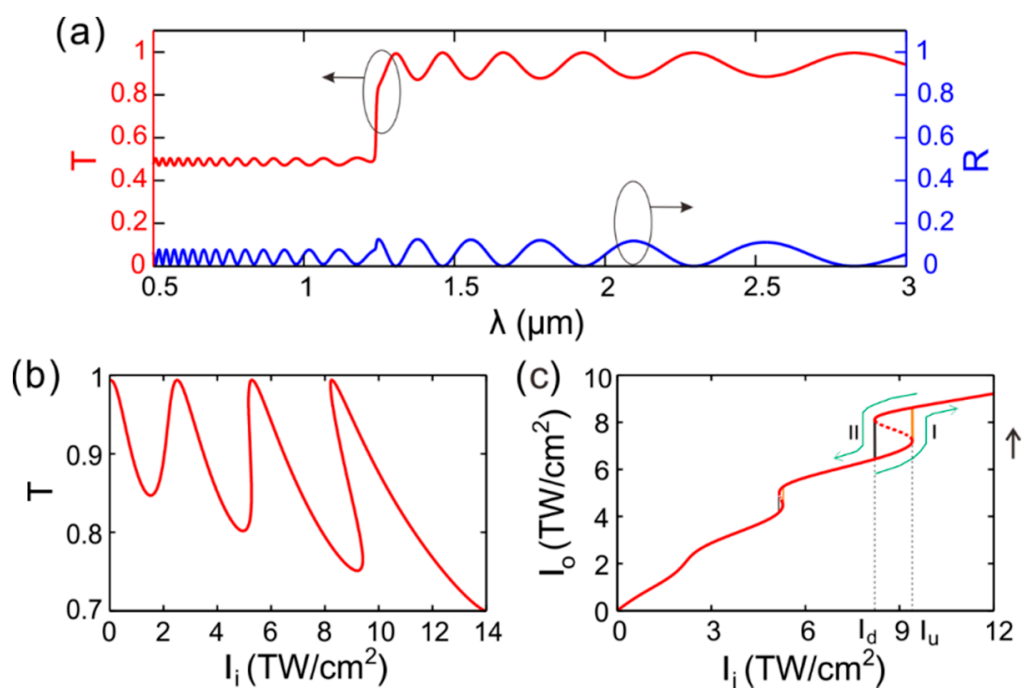

Figure 3. (a) Linear transmission spectrum and reflection spectrum of low-intensity light impinging upon the arrays of graphene sheets. $(\mathbf{b}, \mathbf{c})$ Transmittance and output intensity of light wave changing with the input intensity of light wave, respectively. The wavelength of incident light is $\lambda=1.308 \mu \mathrm{m}$. The chemical potential of graphene is $\mu_{c}=0.50 \mathrm{eV}$.

As the intensity of incident light is strong enough, so that the third-order nonlinear modification to the surface conductivity of graphene reaches the order of linear counterpart, the influence of light intensity on the transmittance and output intensity of light should be considered. For a fixed incident wavelength, Figure $3 \mathrm{~b}$ provides the relationship between transmittance and the intensity of 
incident light. It can be seen that the transmittance oscillates as the input light intensity increases. In addition, the amplitude of oscillation enlarges gradually. Furthermore, with the increase in intensity of incident light, the negative slope of transmittance curve appears, indicating that optical bistability may be achieved.

Considering the nonlinear effect, the intensity of transmitted light increases with the incident intensity. Figure $3 \mathrm{c}$ provides the input-output relationship. Characteristics of this curve manifest so that it is similar to the hysteresis. By increasing the incident intensity of light, the output intensity makes an abruptly upward jump at $I_{u}$, and the input-output curve varies along path I, while the output light intensity has a downward jump at $I_{d}$ as the incident intensity declines, and the input-output curve goes along path II. So $I_{u}$ is known as the upper threshold, and $I_{d}$ is defined as the lower threshold. Therefore, for the fixed incident intensity between the values of $I_{d}<I_{i}<I_{u}$, it corresponds to two stable resonant states $S_{1}$ and $S_{2}$. In general, the output-input intensity relation evolves along path I by increasing the light intensity, while it evolves along path II by decreasing the light intensity. The curve actually corresponds to a double valued function, similar to the hysteresis loop. The two paths represent different physical meanings and here we put them together in the same schematic to make a sharp contrast.

Figure 4a shows the transmission spectra for three different external voltages on graphene. The value of chemical potential depends on the external voltages biased on graphene. The incident wavelength at the saltus step point of transmittance has a red shift as the chemical potential increases. The Fermi level, i.e., the chemical potential, is a critical value to characterize intraband and interband transitions in the energy band. Therefore, the critical incident wavelength triggering the intraband transition decreases as the external voltage on graphene rises.
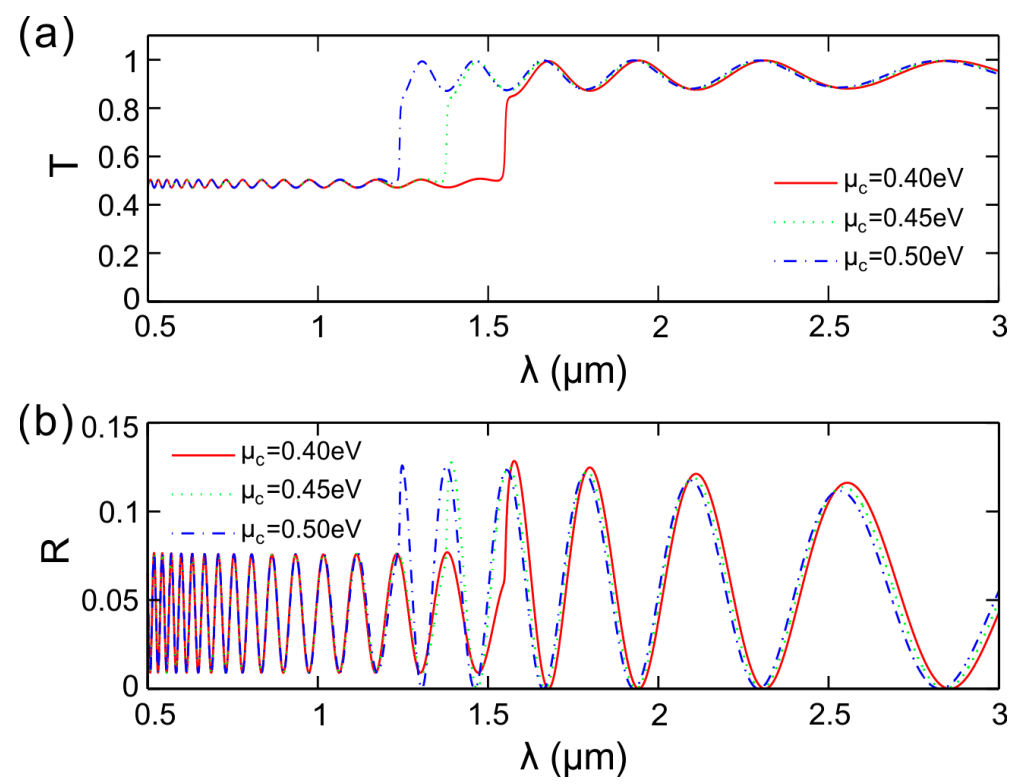

Figure 4. (a,b) Transmission and reflection spectra for different chemical potentials of graphene, respectively.

For different chemical potentials, Figure $4 \mathrm{~b}$ displays their corresponding reflection spectra. One can see that the peaks in reflection spectra are just at the valleys in transmission spectra for a given chemical potential, that is, the maximum transmittance is the conjugate of the minimum reflectivity. For a shorter incident wavelength, interband transitions of electrons in graphene are dominant and graphene can be viewed as metals, so the loss of graphene is greater, and the transmittance is lower. As the incident wavelength increases, intraband transition dominates and the loss of light in graphene decreases. Meanwhile, most of the light has been transmitted. Interband transition only occurs as the incident photon satisfies the condition $\hbar \omega_{1}>2\left|E_{F}\right|$. The electrons emitted by interband transition lead to 
considerable loss of light. Therefore, with the increasing of the chemical potential, the lower limit of frequency of photons required for interband transition increases, which results in a shift in the $R$ and $T$ curves towards lower wavelengths, which could be utilized to modulate the reflectivity and transmittivity of light.

The third-order nonlinearity, known as Kerr effect, needs to be considered as the incident intensity of light is strong enough. The transmittance, therefore, may change with the input intensity of light as a strong laser impinges. Figure 5 a gives the nonlinear transmittance for different values of chemical potential. One can see that, for a given incident intensity, the nonlinear transmittance for $\mu_{c}=0.40 \mathrm{eV}$ is larger than those for $\mu_{c}=0.45$ and $0.50 \mathrm{eV}$. This characteristic results from the fact that the linear transmittance for the chemical potential, $0.40 \mathrm{eV}$, is significantly higher than the two others. With the same incident intensity of light, larger nonlinear transmittance results in stronger transmitted intensity.
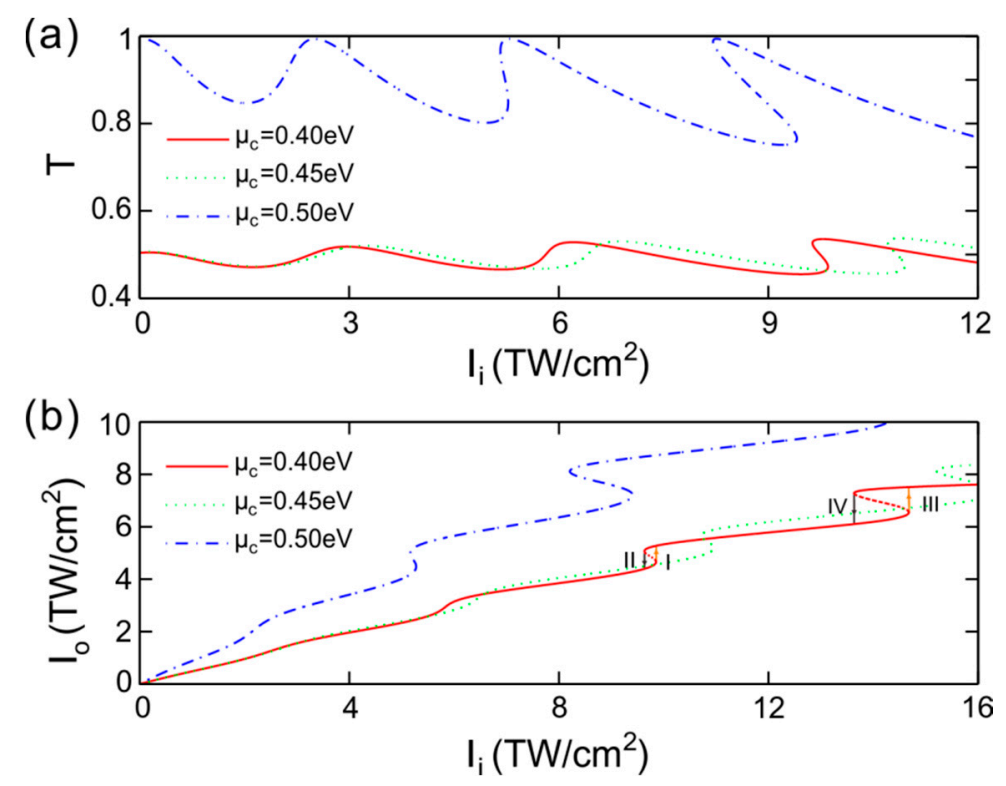

Figure 5. (a,b) Transmittance of light and transmitted intensity versus incident intensity of light for different chemical potentials of graphene, respectively.

The negative value in the curve slope of the nonlinear transmittance indicates that optical bistability may be realized if given an appropriate incident intensity. Figure $5 \mathrm{~b}$ provides the input-output relationship for different chemical potentials. It can be seen that, for $\mu_{c}=0.40 \mathrm{eV}$, two bistablities appear in the curve within a given input intensity interval. As the intensity of incident light increases, there are two upward jumps, namely I and III, while the incident light intensity decreases, there are two downward jumps, namely II and IV. This specialty can be applied to polymorphic switches. The upper and lower thresholds of bistability can be tuned by an external voltage on graphene.

In order to explore the mechanism of formation of these jumps in the transmission spectra, we here investigate the relation between the incident wavelength and the linear surface conductivity of graphene. For three given different chemical potentials $\mu_{c}=0.40,0.45$ and $0.50 \mathrm{eV}$, Figure $6 \mathrm{a}$ gives the real part $\operatorname{Re}\left(\sigma_{g}\right)$ of the surface conductivity of graphene. For shorter incident wavelengths, $\operatorname{Re}\left(\sigma_{g}\right)$ approximates to $6 \mathrm{~S}$ and keeps constant as the wavelength increases. Otherwise, $\operatorname{Re}\left(\sigma_{g}\right)$ suddenly jumps downward as the wavelength is continuing to increase. The real value always is as low as zero by increasing the wavelength sequentially. We here have viewed graphene as a dielectric with an equivalent thickness, so the imaginary part in the refraction index of graphene is the counterpart of $\operatorname{Re}\left(\sigma_{g}\right)$ representing loss or gain of materials. Therefore, larger $\operatorname{Re}\left(\sigma_{g}\right)$ means greater loss and weaker transmitted intensity. 
(a)

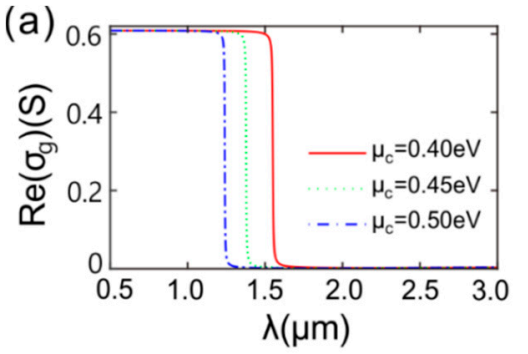

(c)

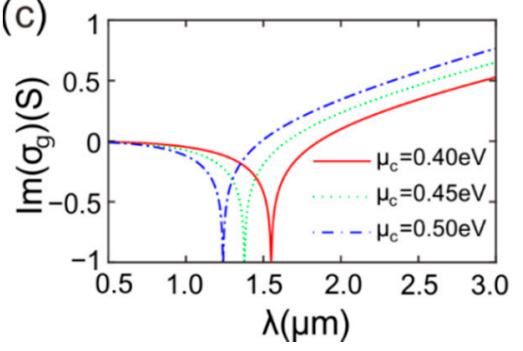

(b)

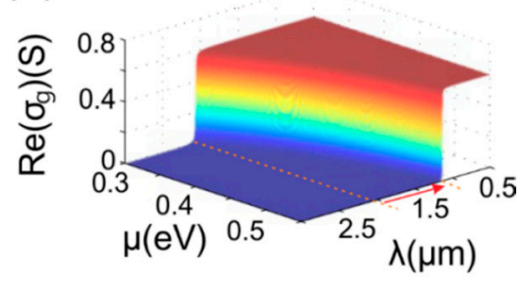

(d)

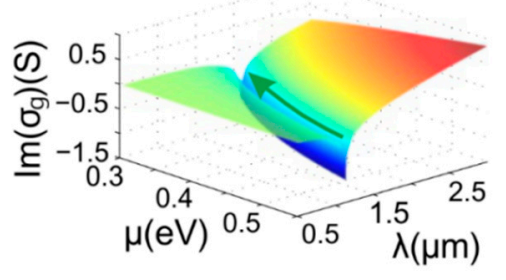

Figure 6. (a,c) $\operatorname{Re}\left(\sigma_{g}\right)$ and $\operatorname{Im}\left(\sigma_{g}\right)$ versus the incident wavelength for some given chemical potentials of graphene, respectively. (b,d) $\operatorname{Re}\left(\sigma_{g}\right)$ and $\operatorname{Im}\left(\sigma_{g}\right)$ changing with the chemical potential and incident wavelength. The red arrow indicates the blueshift of incident wavelength at the saltus step point in surface conductivity as $\mu_{c}$ increases. The green arrow notes the direction of valley shift of $\operatorname{Im}\left(\sigma_{g}\right)$ as $\mu_{c}$ decreases.

Figure $6 \mathrm{~b}$ provides the real part of surface conductivity $\sigma_{g}$ in the parameter space. The chemical potential $\mu_{c}$ in graphene and the incident wavelength compose of the parameter space. It shows that there is a waterfall in $\operatorname{Re}\left(\sigma_{g}\right)$ as the wavelength and the surface conductivity change. The incident wavelength is $\lambda=2.066 \mu \mathrm{m}$ at the saltus step point in the surface conductivity for $\mu_{c}=0.30 \mathrm{eV}$, while the wavelength at the saltus step point is $\lambda=2.066 \mu \mathrm{m}$ for $\mu_{c}=0.55 \mathrm{eV}$. Therefore, the incident wavelength at these points of dramatic change in the surface conductivity is blueshifted as $\mu_{c}$ increases, indicated by the red arrow. The chemical potential can be regulated by an external voltage on graphene. On the other hand, the transmit phase of wave in the arrays of graphene sheets, which can affect transmittance of light, is a function of the surface conductivity. Therefore, the transmittance of light can be flexibly regulated by an external voltage.

The imaginary part $\operatorname{Im}\left(\sigma_{g}\right)$ of the surface conductivity represents the real part of the refraction index of graphene. For three given different values of chemical potential, Figure $6 \mathrm{c}$ demonstrates $\operatorname{Im}\left(\sigma_{g}\right)$ varying with the incident wavelength. There is a sink in each curve and the sink has a blue shift as $\mu_{c}$ increases. However, this obvious feature has a very limited effect on the transmittance due to graphene being ultrathin. It may significantly disturb the complex phase of reflection coefficient.

Figure $6 \mathrm{~d}$ describes the imaginary part of surface conductivity in the parametric space composed of the chemical potential and incident wavelength. There is a groove in the schematic of $\operatorname{Im}\left(\sigma_{g}\right)$ indicated by the arrow. That means the incident wavelength at the valley of $\operatorname{Im}\left(\sigma_{g}\right)$ is redshifted as the chemical potential decreases. Drastic changes in the real index of refraction of equivalent dielectric of graphene have only a slight effect on the transmittance.

If we keep on increasing the incident light intensity, a settled input intensity of light can correspond to several transmittances with a positive slope as shown in Figure 7a. This phenomenon indicates that tristability or multistability could be induced in the input-output relationship as the incident intensity is strong enough. In addition, the nonlinear transmittance may overlap for a stronger incident intensity of light. For the incident wavelength $\lambda=1.308 \mu \mathrm{m}$, we can see that the linear transmittance is larger at $0.5 \mathrm{eV}$ than that at 0.40 or $0.45 \mathrm{eV}$ in Figure $4 \mathrm{a}$. Interband transition dominates for $\hbar \omega_{1}<2\left|E_{F}\right|$, so the chemical potential affects electron transition in graphene. The nonlinear part of the equivalent refractive index of graphene is proportional to the incident intensity of light. A change in refractive index induces phase shifts of the transmission coefficient in the arrays of graphene sheets. Therefore, 
the transmittance varies with the incident intensity and the influence of incident intensity on the transmittance is more obvious for a stronger incident intensity of light.
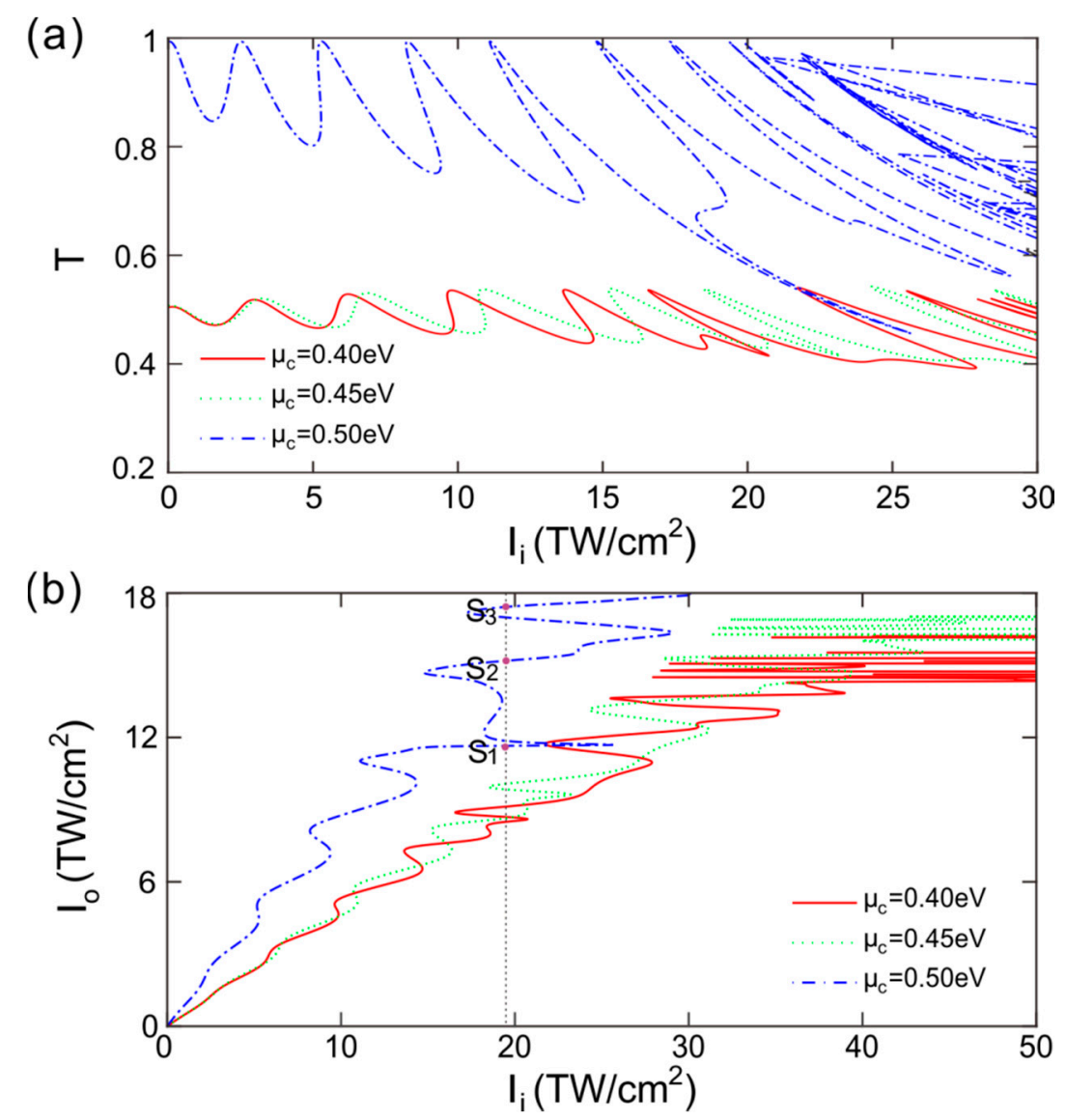

Figure 7. (a) Nonlinear transmittance for optical tristability and multistability. (b) Input-output relation of intensity for optical tristability and multistability.

Figure $7 \mathrm{~b}$ provides optical tristability and multistability for different values of chemical potential. For $\mu_{c}=0.50 \mathrm{eV}$, one can see that the vertical dotted line intersects the input-output curve at three points, i.e., $S_{1}, S_{2}$ and $S_{3}$. This illustrates that for one input there can be three stable outputs, known as optical tristability. As light intensity keeps on increasing, multiple outputs appear for each input, which is known as optical multistability. Optical tristability and multistability may be applied to multivalued all-optical switches or optical storage, respectively. The thresholds and intervals between thresholds of optical tristability and multistability can also be modulated by the external voltage on graphene.

\section{Conclusions}

In conclusion, optical bistability, tristability and multistability in arrays of graphene have been studied in the work. The transmission spectra show there are many resonant modes in this periodic structure and a saltus step point. Optical bistability can be achieved by modulating the input intensity of the light wave or the incident wavelength. The bistable thresholds and the interval between the lower and upper thresholds can also be changed by the external voltage on graphene. Furthermore, optical tristability and multistability are achieved as the intensity of the incident light keeps on increasing. This study may present an application for all-switches, optical storages and logic gates. 
Author Contributions: Conceptualization, X.Z.; Formal analysis, X.K. and D.Z. (Dong Zhong); Funding acquisition, B.X., D.Z. (Dong Zhao) and D.Z. (Dong Zhong); Investigation, X.Z., B.X., M.F. and D.Z. (Dong Zhao); Project administration, X.K.; Resources, X.K.; Software, X.Z., B.X. and D.Z. (Dong Zhao); Supervision, D.Z. (Dong Zhong); Writing—original draft, X.Z., M.F. and D.Z. (Dong Zhao); Writing-review and editing, M.F. All authors have read and agreed to the published version of the manuscript.

Funding: National Natural Science Foundation of China (NSFC) (51975542); the Scientific Research Project of Hubei University of Science and Technology (BK202017, HKCXTD-001); the Science and Technology Plan Research Project of Hubei Education Department (B2019162); and the Industry-University-Research Innovation Foundation of Science and Technology Development Center of Education Ministry of China (2020QT02).

Conflicts of Interest: The authors declare no conflict of interest.

\section{References}

1. Wen, C.P.; Liu, W.; Wu, J.W. Tunable terahertz optical bistability and multistability in photonic metamaterial multilayers containing nonlinear dielectric slab and graphene sheet. Appl. Phys. A 2020, 126, 426. [CrossRef]

2. Nagasaki, Y.; Gholipour, B.; Ou, J.Y.; Tsuruta, M.; Plum, E.; Macdonald, K.F.; Takahara, J.; Zheludev, N.I. Optical bistability in shape-memory nanowire metamaterial array. Appl. Phys. Lett. 2018, 113, 021105. [CrossRef]

3. Rafaie, M.; Hasan, M.H.; Alsaleem, F.M. Neuromorphic mems sensor network. Appl. Phys. Lett. 2019, 114, 163501. [CrossRef]

4. Rahmati, A.; Amandadi, M.A. All-optical NAND/NOR logic gates using bistable switching. Pramana-J. Phys. 2019, 93, 90. [CrossRef]

5. Beeckman, J.; Madani, A.; Vanbrabant, P.; Henneaux, P.; Gorza, S.-P.; Haelterman, M. Switching and intrinsic position bistability of soliton beams in chiral nematic liquid crystals. Phys. Rev. A 2011, 83, 033832. [CrossRef]

6. Afshar, E.N.; Namdar, A. Temperature dependence of the Goos-Hänchen shift in the nonlinear metal-dielectric nanocomposites. Opt. Quant. Electron. 2019, 51, 258. [CrossRef]

7. Schwietert, F.; Kierfeld, J. Bistability and oscillations in cooperative microtubule and kinetochore dynamics in the mitotic spindle. New J. Phys. 2020, 22, 06789. [CrossRef]

8. Barcelos, I.; Maral, L.A.B.; Deneke, C.; Moura, L.G.; Malachias, A. Direct evaluation of CVD multilayer graphene elastic properties. RSC Adv. 2016, 6, 103707-103713. [CrossRef]

9. Jiang, L.; Tang, J.; Xu, J.; Zheng, Z.; Xiang, Y. Graphene tamm plasmon-induced low-threshold optical bistability at terahertz frequencies. Opt. Mater. Express 2019, 9, 139. [CrossRef]

10. Hoang, T.T.; Ngo, Q.M.; Vu, D.L.; Nguyen, H.P.T. Controlling Fano resonances in multilayer dielectric gratings towards optical bistable devices. Sci. Rep. 2018, 8, 16404. [CrossRef]

11. Pan, Z.; Lang, P.; Duan, G. An all-optical switch based on a surface plasmon polariton resonator. Mod. Phys. Lett. B 2018, 32, 1850134. [CrossRef]

12. Liu, F.; Zhao, D.; Cao, H.; Xu, B.; Ke, S. Exceptional points in non-Hermitian photonic crystals incorporated with a defect. Appl. Sci. 2020, 10, 823. [CrossRef]

13. Han, X.; Wang, K.; Xing, X.; Wang, M.; Lu, P. Rabi splitting in a plasmonic nanocavity coupled to a WS monolayer at room temperature. ACS Photonics 2018, 5, 3970-3976. [CrossRef]

14. Fu, N.; Fu, Z.; Zhang, H.; Liao, Q.; Zhao, D.; Ke, S. Topological bound modes in optical waveguide arrays with alternating positive and negative couplings. Opt. Quant. Electron. 2020, 52, 61. [CrossRef]

15. Ke, S.; Zhao, D.; Fu, J.; Liao, Q.; Wang, B.; Lu, P. Topological edge modes in non-Hermitian photonic Aharonov-Bohm cages. IEEE J. Sel. Top. Quantum Electron. 2020, 26, 4401008. [CrossRef]

16. Fu, Z.; Fu, N.; Zhang, H.; Wang, Z.; Zhao, D.; Ke, S. Extended SSH model in non-Hermitian waveguides with alternating real and imaginary couplings. Appl. Sci. 2020, 10, 3425. [CrossRef]

17. Ke, S.; Zhao, D.; Liu, J.; Liu, Q.; Lu, P. Topological bound modes in anti-PT-symmetric optical waveguide arrays. Opt. Express 2019, 27, 13858. [CrossRef]

18. Xu, S.; Zhou, Q.; Zhao, D.; Belić, M.R.; Zhao, Y. Spatiotemporal solitons in cold Rydberg atomic gases with Bessel optical lattices. Appl. Math. Lett. 2020, 106, 106230. [CrossRef]

19. Xu, S.; Li, H.; Qin, Z.; Zhou, G.P.; Zhao, D.; Belić, M.R.; He, J.; Zhao, Y. Parity-time symmetry light bullets in a cold Rydberg atomic gas. Opt. Express 2020, 28, 16322-16332. [CrossRef]

20. Li, L.; Lan, P.; He, L.; Cao, W.; Zhang, Q.; Lu, P. Determination of electron band structure using temporal interferometry. Phy. Rev. Lett. 2020, 124, 157403. [CrossRef] 
21. Yang, Z.; Cao, W.; Mo, Y.; Xu, H.; Mi, K.; Lan, P.; Zhang, Q.; Lu, P. Wave-front controlled attosecond time domain interferometry. arXiv 2019, arXiv:1911.05986. Available online: https://arxiv.org/abs/1911.05986 (accessed on 14 November 2019).

22. Liao, Q.; Cao, W.; Zhang, Q.; Liu, K.; Wang, F.; Lu, P.; Thumm, U. Distinction of electron dispersion in time-resolved photoemission spectroscopy. Phy. Rev. Lett. 2020, 125, 043201. [CrossRef]

23. Pei, C.; Ueda, T.; Zhu, J. Investigation of the effectiveness of graphene/polyvinyl alcohol on the mechanical and electrical properties of cement composites. Mater. Struct. 2020, 53, 66. [CrossRef]

24. Sultana, S.; Bhatti, H.N.; Yasmin, F.; Khan, A.N.; Rehan, I.; Rehan, K.; Noor-ul-Amin. Assessment on the mechanical, structural, and thermal attributes of green graphene-based water soluble polymer electrolyte composites. J. Appl. Polym. Sci. 2020, 137, 48376. [CrossRef]

25. Högl, P.; Frank, T.; Kochan, D.; Gmitra, M.; Fabian, J. Chiral majorana fermions in graphene from proximity-induced superconductivity. Phys. Rev. B 2020, 101, 245441. [CrossRef]

26. Ooi, K.J.A.; Tan, D.T.H. Nonlinear graphene plasmonics. Proc. Math. Phys. Eng. 2017, 473, 20170433. [CrossRef]

27. Lamparski, M.; Van Troeye, B.; Meunier, V. Soliton signature in the phonon spectrum of twisted bilayer graphene. 2D Materails 2020, 7, 2. [CrossRef]

28. Geisenhof, F.R.; Winterer, F.; Wakolbinger, S.; Gokus, T.D.; Durmaz, Y.C.; Priesack, D.; Lenz, J.; Keilmann, F.; Watanabe, K.; Taniguchi, T.; et al. Anisotropic strain-induced soliton movement changes stacking order and band structure of graphene multilayers: Implications for charge transport. Acs Appl. Nano Mater. 2019, 2, 6067-6075. [CrossRef]

29. Liu, Q.; Li, S.; Wang, B.; Ke, S.; Qin, C.; Wang, K.; Liu, W.; Gao, D.; Pierre, B.; Lu, P. Efficient mode transfer on a compact silicon chip by encircling moving exceptional points. Phy. Rev. Lett. 2020, 124, 153903. [CrossRef]

30. Wang, Z.; Wang, B.; Long, H.; Wang, K.; Lu, P. Plasmonic lattice solitons in nonlinear graphene sheet arrays. Opt. Express 2015, 23, 32679. [CrossRef]

31. Massatt, D.; Carr, S.; Luskin, M. Efficient computation of Kubo conductivity for incommensurate 2D heterostructures. Eur. Phys. J. B 2020, 93, 60. [CrossRef]

32. Bao, Q.; Loh, K.P. Graphene photonics, plasmonics, and broadband optoelectronic devices. ACS Nano 2012, 6, 3677. [CrossRef]

33. Hu, B.; Tao, J.; Zhang, Y.; Wang, Q.J. Magneto-plasmonics in graphene-dielectric sandwich. Opt. Express 2014, 22, 21727. [CrossRef]

34. Zhang, R.Z.; Zhang, Z.M. Tunable positive and negative refraction of infrared radiation in graphene-dielectric multilayers. Appl. Phys. Lett. 2015, 107, 948. [CrossRef]

35. Fang, Y.T.; Liang, Z.C. Unusual transmission through usual one-dimensional photonic crystal in the presence of evanescent wave. Opt. Commun. 2010, 283, 2102-2108. [CrossRef]

36. Zhao, D.; Ke, S.; Hu, Y.; Wang, B.; Lu, P. Optical bistability of graphene embedded in parity-time-symmetric photonic lattices. J. Opt. Soc. Am. B 2019, 36, 1731-1737. [CrossRef]

(C) 2020 by the authors. Licensee MDPI, Basel, Switzerland. This article is an open access article distributed under the terms and conditions of the Creative Commons Attribution (CC BY) license (http://creativecommons.org/licenses/by/4.0/). 